\title{
SENTIMIENTO Y AFECTO
}

\author{
FEELING AND AFFECT \\ Silvia Balladares \\ Mario Saiz \\ Universidad Católica del Uruguay
}

\begin{abstract}
Resumen: Se explora la relación de la función sentimiento (Jung, 1921; Millon, 2001), con el afecto positivo y negativo (Watson et al. 1988), y las metas motivacionales expansión -preservación (Millon, 2001), en una muestra de estudiantes universitarios $(n=200)$. La función sentimiento y la expansión - preservación se estudia a través del Inventario de Estilos de Personalidad [MIPS] (Millon, 2001) y el afecto positivo y negativo a través de la Escala de Afecto Positivo y Negativo [PANAS] (Watson, Clark \& Tellegen, 1988), siendo la presente investigación parte de su traducción y adaptación para Uruguay. Los resultados obtenidos muestran que existe una correlación inversa entre la función sentimiento y el afecto positivo, no encontrándose correlación con afecto negativo y tampoco con expansión y preservación.
\end{abstract}

Palabras Clave: función sentimiento; afecto positivo; afecto negativo; psicología analítica; tipos psicológicos

\begin{abstract}
This research explores the relationship between feeling function (Jung, 1921; Millon, 2001) with positive and negative affect (Watson et al. 1988) and the expansion-preservation motivational goals (Millon, 2001) in a sample of 200 college students from Uruguay. The feeling function and expansion-preservation are studied through Millon Inventory of Personality Styles [MIPS] (Millon, 2001) and the positive and negative affect through the Positive and Negative Affect Scale [PANAS] (Watson, Clark \& Tellegen, 1988). The PANAS has recently been translated and adapted to Uruguay. The results show an inverse correlation between feeling function and positive affect. However, the results do not show a correlation with negative affect nor with expansion and preservation.
\end{abstract}

Key Words: feeling function; positive affect; negative affect; analytical psychology; psychological types

\section{Introducción}

La presente investigación explora las relaciones entre la función sentimiento (Jung, 1921/1945) y la dimensión bifactorial de la afectividad: afecto positivo y afecto negativo (Watson, Clark \& Tellegen 1988), con el propósito de contribuir a su discriminación en el abordaje clínico y semántico.

La Psicología Analítica describe las funciones tipológicas como patrones de la conciencia que estructuran la forma de recibir y procesar la información, orientando la relación con el otro, con el mundo y con uno mismo. Esto se logra en base a dos funciones perceptivas: sensación e intuición y dos funciones evaluativas: el pensamiento y el sentimiento (Saiz et al., 2009).

El sentimiento es una de las cuatro funciones adaptativas y evaluadoras de la conciencia, es una función racional - aunque no lógica - y de cualidad personal y subjetiva (Jung, 1969). Conduce a evaluar la cualidad y el valor específico de los objetos. Según Jung (1969), es objetiva- ble con un juicio de agrado o desagrado y con una actitud de rechazo o acercamiento, pero no es reductible a la polaridad placer - displacer. Su juicio genera una memoria sentimental (Von Franz \& Hillman, 1971), que brinda unidad temporal y coherencia interna a las vivencias. "EI sentido del tiempo y del tacto es una tarea del sentimiento que muchas veces es incompatible con la razón del pensamiento" (Von Franz \& Hillman, 1971, p. 133).

La teoría integracionista de Millon (2006), retoma la propuesta tipológica formulada por Jung y la incorpora en la construcción del Inventario de Estilos de Personalidad (MIPS). Las escalas se distribuyen en tres áreas: Metas Motivacionales, Modos Cognitivos y Conductas Interpersonales.

Millon (2006) en la escala del Modo o Estilo Cognitivo, describe un par de bipolaridades entre las cuales se destaca la diferencia entre "... los procesos que se basan esencialmente en el intelecto, la lógica, la razón y la objetividad (reflexión), y los procesos que dependen de la 
empatía afectiva, los valores personales, los sentimientos y la subjetividad (afectividad)" (p. 20). Aclara que el tono emotivo de las palabras transmite más que su contenido o lógica, cuanto más acentuada la tendencia hacia la polaridad sentimiento. Expresa asimismo que “...los sentimientos activarán estados subjetivos tales como unir frente a desunir, sentirse bien frente a sentirse mal, bienestar frente a incomodidad, atracción frente a rechazo, aprecio frente a desprecio, y así sucesivamente" (Millon 2001, p. 40). Una puntuación alta en la escala sentimiento implica que las personas "forman sus criterios según sus propias respuestas afectivas ante las circunstancias, evalúan subjetivamente la repercusión que tendrán sus acciones sobre aquellos que están involucrados y siguen sus metas y valores personales" (Millon 2001, p. 13). En el proceso de evaluar por el sentimiento, la información que proporcionan los sentidos desencadena una respuesta o reacción subjetiva, que puede tener una connotación afectiva neutra, positiva o negativa (Millon, 2001).

En el camino de diferenciar entre sentimiento, emoción, estado de ánimo y evaluaciones afectivas, los aportes de Frijda, Mesquita, Sonnemans y Van Goozen (1991), Ekman (2012) y Rosas (2011), nos posibilitan distinguir sentimientos de otras experiencias afectivas. Rosas (2011) define la especificidad fenomenológica de los sentimientos, argumentando a favor del isomorfismo parcial entre sentimientos y emociones, pero descartando la presunción de que los sentimientos sean epifenómenos de las emociones.

Frijda el al. (1991) discurren sobre la teoría valorativa de las emociones, definiendo los sentimientos como aquella experiencia afectiva, con un carácter de tipo disposicional e intencional, en tanto, es dirigida a un objeto específico y no como una sensación. Ese tipo de intencionalidad sería lo que lo diferencia del estado de ánimo; este último no posa en un objeto intencional preciso, es mas, su causa es vaga y difusa.

Actualmente el sentimiento es definido como disposición emocional o esquema afectivo (Frijda et al., 1991; Ryle, 2009) o como estructura disposicional (Rosas 2011). Se lo diferencia de las emociones (Ekman 2012, Rosas 2011) y del estado de ánimo (Rosas, 2011) tanto por su duración y estabilidad, como por estar dirigido a un objeto intencional específico.

\section{Según Rosas (2011),}

los sentimientos comprenden una visión global y asociativa de sus objetos intencionales, en que la valoración inicial obtenida a través de la emoción, junto a la presencia de pensamientos y recuerdos, genera creencias relevantes tanto para el establecimiento de los sentimientos como disposiciones, como para las pautas de acción subsecuentes (p. 16).

Castilla del Pino (2009), considera los sentimientos como instrumentos que permiten vincularse eficazmente a los objetos mediante un lazo afectivo, permitiendo establecer una organización singular y jerarquizada de los valores.

La tarea instrumental, vinculante y jerárquica de la Función Sentimiento queda de manifiesto luego en sentimientos tales como la pertenencia, la culpa, el respeto, el amor, los celos, la soledad, el vacío, y los sentimientos de sí. Estos pueden sentirse como agradables o desagradables, y también como amenazadores o tranquilizantes y esperanzadores. En todos los casos se diferencian por su fenomenología y características tanto de las emociones, como de los afectos y estados de ánimo.

El estado de ánimo, se define en el DSM IV (APA,1995) como una "...emoción generalizada y persistente que colorea la percepción del mundo" y se describen cinco tipos: el disfórico, elevado, eutímico, expansivo e irritable. Páez y Carbonero (1993) lo caracterizan como "estados afectivos de baja intensidad y relativamente resistentes, con causas antecedentes no inmediatamente salientes, y por lo tanto, con escaso contenido cognitivo; es decir, no tienen una sola causa específica y actúan de forma persistente en el tiempo" (p.134), diferenciándolo así de la emoción. Posteriormente, a partir de sus investigaciones de la expresión facial de las emociones, Ekman (2012) respecto a los estados de ánimo, concluye que no parecerían mostrarse ni en la cara ni en la voz. Su causa difusa, activa emociones concretas y predispone la interpretación del entorno y la respuesta ante las situaciones.

Los resultados de las investigaciones en el dominio de la afectividad hasta la década del 80 no permitían definir, evaluar y establecer claras diferencias entre sentimiento, emoción, y estado de ánimo. Será precisamente a partir de las investigaciones de Zevon y Tellegen (1982), Watson y Tellegen (1985) y Watson et al. (1988), que se obtienen descriptores lo más puros posible del afecto, para poder evaluar el humor o estado de ánimo. Sus investigaciones permiten 
caracterizar la estructura básica del afecto como bidimensional, denominando cada dimensión como afecto positivo y afecto negativo. Cada una de estas dimensiones es relativamente independiente y por tanto no correlacionada, siendo bifactorial a nivel descriptivo. El polo alto representa un estado de alto afecto, y el polo bajo, solo indica la ausencia relativa de implicación afectiva (Watson \& Tellegen, 1985). El afecto positivo (AP) refleja el punto hasta el cual una persona se siente entusiasta, activa, alerta, con energía y participación gratificante. El afecto negativo (AN) representa una dimensión general de distrés subjetivo y participación desagradable que incluye una variedad de estados emocionales aversivos como disgusto, miedo, ira, culpa y nerviosismo.

Watson et al. (1988) validan en Estados Unidos, en una población de $n=4.217$, compuesta por estudiantes universitarios $y$ adultos no universitarios, un cuestionario de autoinforme denominado Positive and Negative Affect Schedule (PANAS), aislando 10 descriptores de afecto positivo (AP) y 10 de afecto negativo (AN); constructos que constituyen los 20 ítems de su versión actual. Watson et al. (1988) comprueban que ambas subescalas del PANAS (AP y AN); poseen niveles adecuados de fiabilidad y validez (factorial convergente y discriminante), manteniéndose las propiedades psicométricas aún cuando se modifiquen las instrucciones temporales de aplicación (estado-rasgo).

La estructura bifactorial del afecto postulada por Watson y sus colaboradores, se ha visto corroborada en diferentes culturas y países, con la traducción y adaptación de PANAS. En los países de habla hispana encontramos las publicaciones de Sandín et al., 1999; Robles y Páez, 2003; Moral, 2011 y Gargurevich y Matos, 2012. En población uruguaya la presente investigación ha contribuido a la traducción y adaptación del PANAS (Saiz, Palacios, Balladares, \& Machín, 2013) brindando así un instrumento valioso tanto para la investigación de la afectividad, como para su aplicación en el campo de la Psicología Cínica.

En el proceso de integración teórica y empírica realizado al crear el instrumento MIPS de medida de la personalidad, Millon (2001) explora las relaciones entre las expresiones afectivas de la bipolaridad placer - dolor y lo que llama el factor de la emocionalidad positiva que fuera descrito por autores como Matson,
Clark y Tellegen (1985; 1988) y Watson y Tellegen (1985). Decide integrar en la escala Metas Motivacionales, el constructo bipolar expansión-preservación, relacionadas a lo que posteriormente se denominó AP y AN. Sería expansión el constructo que compone el concepto de AP, y preservación el que compone AN. Si el supuesto del que parte Millon (2001) se verifica, entonces la correlación entre la escala de metas motivacionales (expansiónpreservación) y modos cognitivos (sentimientopensamiento), es esperable que presente similares características a la correlación existente entre AP y AN con la escala modos cognitivos (sentimiento-pensamiento).

Según Millon (2001), cuando se habla de alta puntuación en expansión, se describe a personas que buscan experiencias y desafíos, a la vez que, son impulsados por el deseo de enriquecer sus vidas. En tanto, cuando se habla de preservación, lo que se muestra es un tendencia a la evitación exitosa de riesgos y peligros que son innecesarios.

La interrogante que persiste es ¿qué relación existe entre el afecto positivo y negativo (Watson et. al. 1988) o entre expansión - preservación (Millon, 2001) y la función sentimiento (Jung, 1969).

El objetivo del presente trabajo es describir las relaciones de la función sentimiento (Jung 1969, Millon 2001) con el afecto positivo y negativo (Watson et al., 1988), así como con expansión - preservación (Millon, 2001), en una población de estudiantes universitarios, reflexionando a partir de los resultados obtenidos acerca de los aportes que podrían ser de interés en el dominio de la afectividad y en la práctica de la Psicología Clínica.

\section{Método}

\section{Participantes}

Se utilizó un muestreo no probabilístico intencional de $\mathrm{N}=200$ participantes, provenientes de instituciones universitarias privadas. La muestra se dividió entre estudiantes de Psicología, un $50.5 \% \quad(n=101)$ y estudiantes de Ciencias Económicas, un $49.5 \%(n=99)$, que se seleccionaron en forma aleatoria.

La población de la muestra se integra con un $71 \%(n=142)$ de individuos de sexo femenino y un $29 \%(n=58)$ de sexo masculino. 
La media de edad es de $23,65(\mathrm{DE}=4,85)$ y el rango de edades se encuentra entre los $18 \mathrm{y}$ 48 años.

En referencia a su lugar de residencia, un $53.5 \%(n=107)$ se radica en la ciudad de Montevideo y un $46.50 \%(n=93)$ en la ciudad de Maldonado, en Uruguay. Un 52\% ( $n=104)$ trabajan mientras cursan sus estudios y un $48 \%$ $(n=96)$ no trabajan.

\section{Instrumentos}

- Inventario Millon de Estilos de Personalidad [Millon Index of Personality Styles por sus siglas en inglés, MIPS], (Millon, 2001). La técnica fue elaborada por Theodor Millon, junto a Lawrence Weiss, Carrie Millon y Roger Davis. Se publica en USA como inventario, con la denominación Millon Index of Personality Styles (MIPS) en 1994. Sus fundamentos teóricos están basados en un modelo de personalidad profundamente arraigado en la teoría biosocial y evolutiva, integrando contribuciones de Freud, Jung, Meyer, Leary y Sullivan. La primera edición en castellano proviene de la traducción y adaptación del instrumento original inglés (Millon, 1994) al español en 1997, publicado en el 2000 por Casullo y Castro, primero en Argentina y posteriormente en España (Sánchez-López \& Aparicio, 1998).

El MIPS está compuesto por 180 ítems que se responden con verdadero o falso. Fue diseñado para medir estilos de personalidad en adultos normales de entre 18 y 65 años de edad. Se incluyen 24 escalas agrupadas en 12 pares y cada par contiene dos escalas yuxtapuestas. A su vez los 12 pares están organizados en tres áreas: Metas Motivacionales, Modos o Estilos Cognitivos y Relaciones Interpersonales. De estas tres áreas, la presente investigación analizará las dos primeras. En el área Metas Motivacionales, respecto a la escala expansión - preservación, se investiga la relación que mantiene con el afecto positivo y el afecto negativo descriptos por Tellegen (1985), Clark \& Watson (1988).

En el área Modos Cognitivos, respecto a la escala pensamiento - sentimiento (Jung 1921/1945) (Millon, 2001) del constructo bipolar intelecto-afecto (Millon, 2001) se investiga el comportamiento del sentimiento como función. Esta escala atiende a los procesos a través de los cuales se recibe, organiza y asimila la información. Se refiere a si esta información desencadena una evaluación reflexiva, lógica, coherente (pensamiento) , o si por el contrario, la evaluación se almacena afectivamente como neutra, positiva o negativa (sentimiento).

Se obtienen puntajes de prevalencia (PP) que indican la presencia o ausencia de la escala medida. Un PP superior a 75 es un coeficiente de confiabilidad que indica presencia del rasgo en estudiantes universitarios (Millon, 1997/2006). Para la corrección del MIPS, se utilizó el soporte informático que se generó a tales efectos.

- Escala de Afecto Positivo y Afecto Negativo [Positive and Negative Affect Schedule por sus siglas en inglés, PANAS],(Watson, Clark, \&Tellegen, 1988). Es un cuestionario de autoinforme de 20 ítems, que consta de dos sub-escalas. Las sub escalas miden el afecto positivo (AP) y el afecto negativo (AN), preguntando al sujeto como se siente y solicitando su valoración en relación a los diez adjetivos o constructos que corresponden al afecto positivo (interesado, fuerte, entusiasmado, orgulloso, excitado, inspirado, decidido, atento, activo, alerta), y al afecto negativo (asustado, disgustado, culpable, atemorizado, hostil, angustiado, nervioso, irritable, avergonzado, inquieto).

El formato de respuesta es de tipo Likert: muy poco o nada (1); algo (2); moderadamente (3); bastante (4); extremadamente (5). La puntuación alcanzada se obtiene sumando los valores de respuesta asignados a cada ítem de cada una de las escalas respectivamente. En nuestro caso, de los dos períodos de tiempo evaluados por el PANAS: estado (referidos a como se ha sentido en la última semana) y rasgo (referido a como se siente generalmente), se seleccionó rasgo, dado que permite apreciar el estado de ánimo con mejor nivel de discriminación de la emoción $y$ el sentimiento.

La presente investigación contribuye al primer estudio de traducción y adaptación de la escala PANAS al español para Uruguay (Saiz, Palacios, Balladares \& Machín, 2013). En primer lugar se desarrolla el proceso de traducción retrotraducción (back-translation) y adaptación de la escala (Nunnally \& Bernstein, 1995). En segundo lugar se evalúan las propiedades psicométricas del PANAS, en una muestra de estudiantes uruguayos $(n=200)$, con la expectativa de encontrar resultados similares al reporte original de validación.

El examen de la consistencia interna del instrumento resulto aceptable en términos de fiabilidad, entendida como la exactitud o precisión de una medición, o el grado en que 
las puntuaciones del test están libres de errores de medición (Tornimbeni, Pérez \& Olaz, 2008). El procedimiento utilizado para la estimación del coeficiente de confiabilidad, fue el Alfa de Cronbach que presenta en la subescala del afecto negativo un valor de 0,77 en la modalidad temporal rasgo-generalmente y de 0,76 en la modalidad estado-última semana. En la subescala afecto positivo presento un valor de 0,79 (rasgo-generalmente) y 0,86 (estado-última semana). Esto sugiere que ambas escalas muestran un grado adecuado de homogeneidad entre los ítems, lo cual aumenta la precisión del instrumento (Cohen \& Swerdlik, 2006). Las escalas de afecto positivo y negativo demostraron ser confiables. De esta manera tuvimos evidencia de la presencia de dos factores que representan el afecto positivo y negativo (Watson et al., 1988). El análisis factorial (AFE) mostró la agupación de ítems en dos factores luego de aplicar rotación ortogonal Varimax para las dos modalidades: rasgo-generalmente y estado-última semana, mostrando coherencia con la estructura teórica del PANAS.

En el estudio del coeficiente de correlación de Pearson se observa que dos ítems "alerta" e "inquieto" tenían un $r<0,40$ por lo cual se plantea su seguimiento en el Análisis Factorial Exploratorio (AFE). Los resultados muestran que ellos merecen una consideración aparte, porque a excepción de ellos, todos los demás ítems de la escala PANAS presentaron saturación factorial coherente y con claro significado teórico.

Por tanto, se ha presentado en este estudio una versión delPANAS adaptadaal español deUruguay.

\section{Procedimiento y análisis de datos}

Los instrumentos PANAS y MIPS se aplican a la muestra de estudiantes universitarios en forma auto administrada y colectiva, en sus centros de estudio. Los participantes firman voluntariamente un consentimiento informado aceptando ser parte de la investigación. Los datos recogidos son cargados y analizados en el paquete SPSS 21 , comenzando en primer lugar con un análisis descriptivo de los mismos, incluyendo los resultados de la media $(\dot{x})$ y la desviación típica $(\sigma)$.

En segundo lugar, se explora la presencia de correlación entre afecto positivo y afecto negativo (PANAS), en su versión rasgo-generalmente y el tipo psicológico sentimiento (MIPS).

En tercer lugar, se explora la correlación entre afecto positivo y negativo (PANAS) en su versión rasgo-generalmente, con preservación y expansión (MIPS).

Para estudiar en ambos casos la correlación se calcula el coeficiente de correlación de Pearson. Para calificar el grado de asociación entre las variables estudiadas se utiliza el criterio de correlación sugerido por Morales Vallejo ( 2008). La escala de correlación lineal bilateral propuesta describe la correlación como muy baja (0 y .20), baja (.20 y .40), moderada (.40 y $.60)$, apreciable, más bien alta (.60 y .80), alta o muy alta $(.80$ y 1$)$.

Finalmente, se evalúa la fiabilidad mediante la consistencia interna de cada escala medida por el coeficiente alfa de Cronbach.

\section{Resultados}

En la muestra de población estudiada $(n=200)$ encontramos que el modo cognitivo más utilizado para procesar la información es la función sentimiento, presentando una media sensiblemente mayor que la función pensamiento. Esto indica que entre estudiantes universitarios de la muestra, es mayor la tendencia a registrar lo que sienten en relación con las cosas y no lo que piensan acerca de las mismas, Tabla 1.

\section{Tabla 1}

Valores de la media y desviación típica para afecto positivo, negativo y las funciones tipológicas de sentimiento y pensamiento $(n=200)$

\begin{tabular}{lcc}
\hline & Media & Desviación típica \\
\hline Sentimiento & 64,83 & 24,748 \\
Pensamiento & 43,61 & 27,167 \\
AP & 33,51 & 5,537 \\
AN & 20,19 & 5,688 \\
\hline
\end{tabular}

Según surge de la Tabla 2, la escala sentimiento como función superior (sentimiento $\geq$ pensamiento, sensación e intuición), se presenta en un $44 \%(n=88)$ de los casos analizados, siendo significativamente mayor en personas de sexo femenino.

Tabla 2

Función sentimiento como función superior, por sexo

\begin{tabular}{lcccccc}
\hline & \multicolumn{2}{c}{ Mujeres } & \multicolumn{3}{c}{ Hombres } & \multicolumn{2}{c}{ Total } \\
& $\%$ & $\mathrm{n}$ & $\%$ & $\mathrm{n}$ & $\%$ & $\mathrm{n}$ \\
\hline $\begin{array}{l}\text { Función Sup. } \\
\text { Sentimiento }\end{array}$ & 40 & 80 & 4 & 8 & 44 & 88 \\
$\begin{array}{l}\text { Función } \\
\text { Sentimiento } \geq 75\end{array}$ & 30.5 & 61 & 3 & 6 & 33.5 & 67 \\
\hline
\end{tabular}


El modo cognitivo sentimiento es el preferido para procesar la información, presentándose como función superior con un puntaje de prevalencia $\geq 75$, en un $33.5 \%(n=67)$ de los casos. Esto reafirma la confiabilidad en la medición de la variable analizada. Asimismo es mayor su prevalencia entre quienes estudian Psicología (30\%), comparativamente con quienes estudian Ciencias Económicas (14\%), Tabla 3.

Tabla 3

Función sentimiento como función superior, por formación

\begin{tabular}{lcccccc}
\hline & \multicolumn{2}{c}{ Psicologia } & \multicolumn{2}{c}{ Cs. Económicas } & \multicolumn{2}{c}{ Total } \\
& $\%$ & $\mathrm{n}$ & $\%$ & $\mathrm{n}$ & $\%$ & $\mathrm{n}$ \\
\hline Función & 30 & 60 & 14 & 28 & 44 & 88 \\
$\begin{array}{l}\text { Sup. } \\
\text { Sentimiento }\end{array}$ & & & & & & \\
$\begin{array}{l}\text { Función } \\
\text { Sentimiento } \\
\geq 75\end{array}$ & 25 & 50 & 8.5 & 17 & 33.5 & 67 \\
\hline
\end{tabular}

En relación a la función pensamiento (modo cognitivo) como función superior, se siguió el mismo criterio de análisis (pensamiento $\geq 75$ ), resultando que un $11.5 \%(n=23)$ procesa la información interpretando la realidad a partir de datos objetivos e impersonales, producto del pensamiento lógico en primer lugar, Tabla 4. En el comportamiento de la prevalencia de ambas variables, por una parte la función sentimiento y por otra el afecto (positivo y negativo), se encuentran diferencias significativas que ratifican la discriminación entre ambos constructos. Existe una marcada preponderancia del afecto positivo, frente a quienes presentan afecto negativo, a diferencia de los resultados obtenidos por la función sentimiento.

Tabla 4

Prevalencia de las variables en la muestra estudiada $(n=200)$

\begin{tabular}{lcc}
\hline & $\%$ & $\mathrm{n}$ \\
\hline Sentimiento & 33.5 & $67^{*}$ \\
Pensamiento & 11.5 & $23^{\star *}$ \\
AP & 95.50 & 189 \\
AN & 5.50 & 11 \\
\hline
\end{tabular}

* P6B.PP $\geq 75$ y $\geq$ P6A.PP ; P5A.PP ; P5B.PP.Tipo sentimiento como función superior

** P6A.PP $\geq 75$ y $\geq$ P6B.PP ; P5A.PP ; P5B.PP.Tipo pensamiento como función superior

Al analizar la relación existente entre el sentimiento y el afecto (positivo y negativo), encontramos que el sentimiento correlaciona en forma inversa y negativa muy baja con el afecto positivo $(r=-.18)$. También que no correlaciona con afecto negativo ( $r=.01)$, según se muestra en la Tabla 5. Respecto al pensamiento, observamos que correlaciona en forma positiva con AP $(r=.18)$ y en forma positiva también con AN ( $r=.17)$.

Tabla 5

Correlación entre Afecto Positivo y Negativo (PANAS) y las funciones tipológicas Pensamiento - Sentimiento (MIPS) ( $n=200)$

\begin{tabular}{lcc}
\hline Modos cognitivos & $\mathrm{AN}(\mathrm{n}=11)$ & $\mathrm{AP}(\mathrm{n}=189)$ \\
\hline Sentimiento & $.17^{*}$ & $.18^{* *}$ \\
Pensamiento & .01 & $-.18^{*}$ \\
\hline
\end{tabular}

** La correlación es significativa al nivel 0,01 (bilateral)

* La correlación es significante al nivel 0,05 (bilateral)

En la Tabla 6 se presentan los resultados obtenidos, encontrando entre AP y Expansión una correlación baja $(r=.27)$, aunque estadísticamente significativa (positiva). En cuanto a AN y Preservación, encontramos una correlación moderada $(r=.44)$ y estadísticamente significativa (positiva). Respecto a la correlación entre AP y Preservación ( $r=-.25)$, y entre AN y Expansión $(r=-.40)$, ambas son inversas o negativas, siendo baja y moderada respectivamente.

Tabla 6

Correlación entre Afecto Positivo y Negativo (PANAS) y Modos Motivacionales Expansión - Preservación (MIPS) $\quad(n=200)$

\begin{tabular}{lcc}
\hline $\begin{array}{l}\text { Metas } \\
\text { Motivacionales }\end{array}$ & A N $(\mathrm{N}=11)$ & A P $(\mathrm{N}=189)$ \\
\hline Expansión & $-.40^{* *}$ & $.27^{* *}$ \\
Preservación & $.44^{* *}$ & $-.25^{* *}$ \\
\hline \multirow{2}{**}{ La correlación es significativa al nivel 0,01 (bilateral) } \\
* La correlación es significante al nivel 0,05 (bilateral)
\end{tabular}

En la Tabla 7 se muestra la correlación entre sentimiento y expansión. Encontramos que no existe correlación ( $r=-.01$ ) entre ambas. En el caso del pensamiento hay una correlación estadísticamente significativa aunque muy baja $(r=.14)$ con Preservación.

Tabla 7

Correlación entre Modo Cognitivo Sentimiento y Meta Motivacional Expansión -Preservación (MIPS) $(n=200)$

\begin{tabular}{lcc}
\hline Modos cognitivos & $\begin{array}{c}\text { Expansión } \\
\text { (Meta } \\
\text { Motivacional) }\end{array}$ & $\begin{array}{c}\text { Preservación } \\
\text { (Meta }\end{array}$ \\
\hline Pensamiento & -.00 & $.14^{*}$ \\
Sentimiento & -.01 & .10 \\
\hline
\end{tabular}

** La correlación es significativa al nivel 0,01 (bilateral)

* La correlación es significante al nivel 0,05 (bilateral)

En la Tabla 8 se muestra la correlación de sentimiento y pensamiento con los constructos del afecto (AP, AN, Expansión y Preservación). La correlación entre sentimiento y AP es estadísticamente significativa, aunque muy baja y de carácter negativo e inverso $(r=-.18)$. 
En cambio, pensamiento correlaciona con AN $(r=.17)$, AP $(r=.18)$ y Preservación $(r=.14)$, en forma positiva y estadísticamente significativa, aunque muy baja.

Tabla 8

Correlación entre Pensamiento y Sentimiento (Modos cognitivos) y AN-Preservación y AP- Expansión $(n=200)$

\begin{tabular}{ccccc}
\hline $\begin{array}{c}\text { Modos } \\
\text { cognitivos }\end{array}$ & $\begin{array}{c}\text { Afecto } \\
\text { Negativo } \\
\text { (AN) }\end{array}$ & $\begin{array}{c}\text { Afecto } \\
\text { Positivo } \\
\text { (AP) }\end{array}$ & $\begin{array}{c}\text { Preservación } \\
\text { (Meta } \\
\text { Motivacional) }\end{array}$ & $\begin{array}{c}\text { Expansión } \\
\text { (Meta } \\
\text { Motivacional) }\end{array}$ \\
\hline Pensamiento & $.17^{*}$ & $.18^{* *}$ & $.14^{*}$ & -.00 \\
Sentimiento & .01 & $-.18^{*}$ & .10 & -.01 \\
\hline
\end{tabular}

** La correlación es significativa al nivel 0,01 (bilateral)

* La correlación es significante al nivel 0,05 (bilateral)

\section{Discusión}

Se considera importante señalar que no se han encontrado investigaciones previas que indaguen las relaciones entre la función sentimiento y el afecto positivo y negativo. La presente investigación pretende contribuir tanto al conocimiento específico de cada una, encontrando posibles correlaciones, así como en la discriminación entre ambas de acuerdo al comportamiento observado.

EI instrumento PANAS en su proceso de traducción y adaptación para Uruguay ha demostrado un buen comportamiento psicométrico y adecuación al constructo teórico que valora. Los resultados han sido similares a los observados en estudios antecedentes en la lengua española (Sandín et al. 1999; Robles \& Páez, 2003; Gargurevich \& Matos, 2012; Moriondo, De Palma, Medrano \& Murillo 2012), que muestran que el PANAS tiene una estructura teórica bifactorial. Tales características lo convierten en un instrumento inicialmente válido y confiable, para ser usado tanto en investigación como en clínica en la población de adultos estudiados en nuestro país.

Respecto a los resultados obtenidos a partir de la muestra estudiada $(n=200)$, se encuentra que un $95.5 \%(n=189)$ de los individuos presenta afecto positivo (AP), caracterizado por sentirse entusiasta, activo, alerta, decidido, con fortaleza y aumento del interés, con energía y participación gratificante, orgullo e inspiración. Asimismo un 5.5. $\%(n=11)$ presenta afecto negativo (AN), caracterizado por desinterés subjetivo y participación desagradable que incluye una variedad de estados emocionales aversivos como disgusto, ira, culpa, miedo y nerviosismo (Watson et al., 1988).
En cuanto al análisis de la función sentimiento, ésta se presenta como función superior o prevalente (sentimiento $\geq$ pensamiento, sensación e intuición) en el $44 \%(n=88)$ del total de los casos. La función superior, según explica la Psicología Analítica es aquella función más desarrollada y discriminada (Von Franz \& Hillman, 1971) que organiza y orienta la actitud del individuo. Millon (2001) expresa que en estos casos la evaluación subjetiva se realiza en base a valores y metas personales que determinan la respuesta afectiva, pudiendo entonces estar ante una "actitud disposicional" de la función racional. En un $33.5 \%(n=67)$ del total de los casos, estamos además ante un puntaje elevado y significativo ( $\geq 75$ ), distribuido en un $30.5 \%$ de sexo femenino y un $3 \%$ de sexo masculino, así como el $25 \%$ son estudiantes de Psicología y el $8,5 \%$ de Ciencias Económicas. Del $44 \%$ tipo sentimiento, un $75.5 \%(n=46)$ son del tipo sentimiento-intuición y un $23.9 \%(n=14)$ del tipo sentimiento - sensación.

Observamos, en base a los resultados obtenidos, que existe un comportamiento diferente de ambas variables (afecto y sentimiento) en una misma muestra de población $(n=200)$. En este sentido, el $95.5 \%$ presenta generalmente afecto positivo, mientras el $33.5 \%$ presenta sentimiento como función superior, con un puntaje elevado y significativo. Un primer análisis conduce al punto de inflexión a partir del cual Jung (1969) y posteriormente Millon (2001), conciben el sentimiento como una función tipológica de la conciencia diferente del afecto a nivel estructural y psicodinámico. Reconocemos que la correlación entre dos variables es relativa a los instrumentos utilizados (Morales Vallejo 2008). No puede establecerse una causalidad o relación directa entre ambas variables, pero los resultados nos invitan a focalizar en la correlación encontrada.

Se analiza entonces la correlación bilateral entre la función sentimiento y el afecto positivo, encontrando que es estadísticamente significativa e inversa $(r=-18)$. Esto nos indica que cuando una aumenta la otra disminuye. Entonces un individuo con afecto positivo elevado vería disminuida su capacidad para valorar a través de la función sentimiento o si aumenta la actividad de la función sentimiento, disminuye la presencia del afecto positivo. Para ilustrar los resultados diríamos que cuanto mayor es el entusiasmo (descriptor de AP) o el interés (descriptor de AP) del individuo, menor sería su capacidad para valorar a través de la función sentimiento 
como agradable o desagradable, o para tomar posteriormente una actitud racional de rechazo o acercamiento.

La estructura bifactorial del afecto conduce a analizar la dimensión del afecto negativo en forma relativamente independiente. No se encontró correlación entre el afecto negativo y la función sentimiento ( $r=.01$ ), lo que invita a la reflexión sobre sus implicaciones clínicas.

En el orden de la especificidad fenomenológica de la función sentimiento, ésta se discrimina del afecto positivo y negativo.

Esta discriminación presente en el MIPS en la diferencia entre el modo cognitivo pensamiento - sentimiento y las metas motivacionales expansión - preservación, se profundiza en el paralelismo planteado por Millon (2001) dentro del constructo bipolar placer -dolor, entre expansión (meta motivacional) y afecto positivo, así como entre preservación (meta motivacional) y afecto negativo. Se analiza entonces esta correlación a efectos de verificar dicha hipótesis. Los resultados indican respecto a expansión, una correlación estadísticamente significativa con afecto positivo $(r=+.27)$ y moderada e inversa con AN ( $r=-.44)$. Respecto a la correlación entre preservación y afecto negativo, se confirma que es estadísticamente significativa $(r=+.44)$, así como baja e inversa con AP $(r=-.25)$. Su cruzamiento sostiene la condición bifactorial y bidimensional entre AP y preservación así como entre AN y expansión. Este hallazgo adviene desde los instrumentos PANAS y MIPS a una analogía factible entre los constructos bipolares del placer-dolor (modos motivacionales expansión - preservación) y la estructura bidimensional del afecto (positivo-negativo). Se confirma la hipótesis de Millon (2001) por la cual había integrado la escala metas motivacionales asimilando la expansión al afecto positivo y la preservación al afecto negativo, ambas dentro del constructo bipolar placer - dolor.

En función de esta analogía, se explora la correlación entre expansión - preservación (modo motivacional) con la función sentimiento, encontrando que no es estadísticamente significativa.

Los resultados de la presente investigación reafirman la diferencia en el comportamiento psicodinámico de los constructos afecto y función sentimiento. Esto contribuye a su discriminación e implica que tanto en desarrollos teóricos como técnico-instrumentales, su utilización como sinónimos o denominaciones intercambiables sería incorrecta.
Autores contemporáneos (Jung 1969, Millon 2001, Ekman 2012, Rosas 2011, Castilla del Pino 2009) discriminan el estado de ánimo (afecto positivo y negativo) de la función sentimiento y de la emoción, a partir de criterios como duración, estabilidad y dirección, de lo específico o no de su objeto intencional y de su relación con la fijación de creencias generadoras de emociones subyacentes (Rosas 2011). Sus diferencias pueden ser observadas también a través de la presencia o ausencia de la expresión facial de las mismas (Ekman, 2012). Estos criterios se encuentran vehiculizando puentes en la actualidad que re-significan sus funciones en el proceso evolutivo del desarrollo de la personalidad, así como en el comportamiento y semiología clínica. Los resultados concuerdan con la concepción psicodinámica de Jung (1969) y posteriormente de Millon (2001), respecto al componente disposicional de la función sentimiento, que Rosas (2011) reafirma definiéndola como una estructura disposicional dirigida a un objeto intencional específico, diferenciándola a su vez del estado de ánimo.

\section{Referencias}

American Psychiatric Association (1995). Manual Diagnóstico y Estadístico de los Trastornos Mentales, DSM VI. Barcelona: Masson S.A. Carretero-Dios, H., \& Perez, C. (2005). Normas para el desarrollo y revisión de estudios instrumentales. International Journal of Clinical and Health Psychology, 5 (3), pp. 521-551.

Castilla del Pino, C. (2009). Teoría de los sentimientos. (4. ${ }^{a}$ ed.) Barcelona: Fabula Tusquets

Casullo M. M., \& Castro Solano A. (2000). Adaptación del MIPS en Argentina. En M.P. Sánchez - López y M.M. Casullo. Estilos de Personalidad: Una perspectiva Iberoamericana. Madrid: Miño y Dávila.

Clark L. A., \& Watson D. (1988). Mood and the mundane: Relations between daily life events and self-reported mood. Journal of Personality and Social Psychology, 54, pp. 296-308.

Cohen, R., \& Swerdlik, M. (2006). Pruebas y Evaluación Psicológicas. (6 ${ }^{a}$ ed.) España: Mc. Graw Hill Interamericana.

Ekman, P. (2012). El rostro de las emociones. (2 a ed.). Barcelona: RBA.

Emde, R. (1998). Yendo hacia adelante: Las influencias integradoras de los procesos afectivos en el desarrollo y en el psicoanálisis. Revista APdeBA, XX (3), pp. 473-516.

Frijda N, Mesquita B., Sonnemans J., \& Van Goozen S. (1991). The Duration of Affective Phenomena or Emotions, Sentiments and Passions. International Review of Studies on Emotion. 1, pp. 187-225

Gargurevich R., \& Matos L. (2012). Validez y confiabilidad de escala de afecto positivo y negativo (SPANAS) en estudiantes universitarios peruanos. Psicol., 14 (2), pp. $208-217$. 
Green, A (1973). El discurso vivo. Una concepción psicoanalítica del afecto. Valencia: Promolibro.

Green, A (1998). Acerca de la discriminación e indiscriminación afecto-representación. Revista APdeBA, $X X$ (3), pp. 517-587.

Grinberg, Luiz P. (2003). Jung O Homem Criativo, Sao Paulo: FTD.

Hillman, J (2005). El pensamiento del corazón (3a. reimpresión). España: Siruela.

Jung, C. (1921/1945). Tipos Psicológicos. Buenos Aires: Latinoamericana.

Jung, C. (1969). Los Complejosyellnconsciente. Madrid:Alianza.

Jung, C. (1998). Símbolos de Transformación (4a. reimpresión). Barcelona. Paidós Ibérica S.A.

Leivi, B.M. (1998). El inconsciente y los afectos. Revista APdeBA, XX (3), pp. $651-681$.

Lerner, H. (1998)Afectos, afecciones, afectaciones. Revista APdeBA, XX (3), pp. $683-704$.

López, A. ; Rondon, J. ; Alfano, S., \& Cellerino, C. (2012). Relaciones entre esquemas tempranos inadaptados y afectividad positiva y negativa. Ciencias Psicológicas; VI (2), pp.149-173.

Marina, J.L., \& López, M. (1999/2011). Diccionario de los sentimientos. ( $5^{\mathrm{a}}$ ed.) Barcelona: Anagrama.

Millon, Theodore. (2001): MIPS. Inventario de Estilos de Personalidad. Manual Madrid: Tea.

Millon, Theodore. (2006) MIPS. Inventario Millon de Estilos de Personalidad. ( $2^{\text {a }}$ reimpresión). Buenos Aires: Paidós.

Moral J. (2011). La escala de afecto positivo y negativo (PANAS) en parejas casadas mexicanas. Ciencia Ergo Sum, 18 (2), pp. 117 - 125.

Morales Vallejo, P. (2008). Estadística Aplicada a las Ciencias Sociales. España: Pujol \& Amado.

Moriondo M., De Palma P, Medrano L., \& Murillo P. (2012). Adaptación de la Escala de Afectividad Positiva y Negativa a la población de adultos de la ciudad de Córdoba: análisis psicométricos preliminares. Universitas Psychologica, 11 (1), pp. 187 - 196.

Nunnally J. C., \& Bernstein I.J. (1995). Teoría Psicométrica. Madrid: McGraw-Hill.

Paez, D., \& Carbonero, A. (1993). Afectividad, cognición y conducta social. Psicothema, 5, pp.133-150.

Rayle G. (2009). The Concept of Mind .Londres: Routledge.

Robles R., \& Paez F. (2003). Estudio sobre la traducción al español y las propiedades psicométricas de las escalas de afecto positivo y negativo (PANAS). Salud Mental; 26 (1), pp. $69-75$.

Rosas, O. (2011). La estructura disposicional de los sentimientos. Ideas y valores, 145 pp. 5 - 31.

Saiz M., Botelho C., Galiás I., de Souza Vargas N., Alves de Araújo C., Arancibia M., ..., Perissinoto J. (2009) Psicopatología Psicodinámica Simbólico-Arquetipica 2. Montevideo: Prensa Médica Latinoamericana.
Saiz M. ,Palacios T., Balladares S., \& Machín D. (2013, octubre). Traducción y Adaptación de la Escala de Afecto Positivo y Negativo (PANAS) en Estudiantes Universitarios en Uruguay. Análisis Psicométricos Preliminares. En IV Simposio de Investigación en Psicología. Posibilidades y Realidades de Innovación. Montevideo: UCU (en prensa).

Sánchez - López M.P., \& Aparicio García M. (1998). El Inventario Millon de Personalidad : su fiabilidad y validez en España y Argentina. Revista Iberoamericana de Diagnóstico y Evaluación psicológica, 4 (2), pp. 87- 110.

Sánchez Lopez M.P., Díaz J., \& García A. (2001). MIPS Inventario de Estilos de Personalidad de Millon. Adaptación Española. Madrid. TEA.

Sandín B., Chorot P., Lostao L., Joiner T., Santed M., \& Valiente R. (1999). Escalas PANAS de afecto positivo y negativo: validación factorial y convergencia transcultural. Psicothema; 11 (1), pp. 37-51.

Tellegen A. (1985). Structures of mood and personality end their relevance to assessing anxiety, with emphasis on self-report. En A.H.Tuma y D. Maser (Eds.) Anxiety and the anxiety disorders. Hillsdale, NJ: Erbaum, pp. 21-49.

Tornimbeni S., Pérez E., \& Olaz F. (2008). Introducción a la Psicometría. Buenos Aires: Paidós.

Von Franz, Marie-Louise \& Hillman, J. (1971): A Tipología de Jung, Sao Paulo: Cultrix.

Watson, D. (in press.) (1988) The vicissitudes of mood measurement: Effects of varying descriptors, time frames, and response formats on measures of Positive and Negative Affect. Journal of Personality and Social Psychology, 55 (1), pp 128-41.

Watson, D. (1988). Intraindividual and interindividual analyses of Positive and Negative Affect: Their relation to health complaints, perceived stress, and daily activities. Journal of Personality and Social Psychology, 54 (6), pp. 1020 - 30.

Watson D., \& Clark L. A. (1984). Negative Affectivity: The disposition to experience aversive emotional states. Psychological Bulletin, 96 (3), pp. 465-490.

Watson, D., Clark, L. A., \& Tellegen A. (1984). Cross-cultural convergence in the structure of mood: A Japanese replication and a comparison with U.S. findings. Journal of Personality and Social Psychology, 47 (1), pp. 127-144.

Watson D., Clark L.A., \& Tellegen A. (1988) Development and validation of brief measures of Positive and Negative Affect: The PANAS scales. Journal of Personality and Social Psychology, 54 (6), pp. 1063 - 70.

Watson, D., \& TellegenA. (1985) Toward a consensual structure of mood. Psychological Bulletin, 98 (2), pp. 219 - 235.

Zevon M., \& Tellegen A. (1982). The structure of mood change: An idiographic/nomothetic analysis. Journal of Personality and Social Psychology 43. pp. 111-122.

Para citar este artículo:

Balladares, S., \& Saiz, M. (2015). Sentimiento y Afecto. Ciencias Psicológicas 9(1): 63 - 71 